\title{
Comments on Monojets and Other Anomalous Events ${ }^{a}$
}

\author{
G. L. KANE \\ Randall Physics Laboratory \\ University of Michigan \\ Ann Arbor, Michigan 48109
}

\section{INTRODUCTION}

The Standard Model (SM) is increasingly well tested and it continues to describe all experiments to the present limits of accuracy. Most particle physicists hope that phenomena will be found that do not fit the SM predictions, not because it will contradict the SM (which is now so well tested that it must be a good description of nature on the $100 \mathrm{GeV}$ scale), but because it will provide clues to physics beyond the SM. There are still many questions whose answers we would like to have, such as why the SM takes the form it does, what is the origin of mass and flavor, can all forces be unified, is supersymmetry (which is an allowed symmetry in a quantum field theory) realized in nature, and more. Even most theorists feel that experimental clues may be needed to untangle these questions or at least will greatly speed up the process.

In recent years, a number of possible effects beyond the SM have been reported, such as Higgs boson candidates with properties inconsistent with a single neutral SM Higgs boson, too large a cross section for production of like-sign dileptons in $\nu$ reactions, several categories of events in the UA1 and UA2 detectors at the CERN collider, and others. Unfortunately, most of them have not stood up to subsequent experimental examination. Even the long-standing like-sign dileptons are not appearing in recently improved experiments. ${ }^{1}$ As we have heard ${ }^{2.3}$ at this meeting, of all the candidates at the CERN collider, only the UA1 monojet events (and perhaps some associated dijet events with missing momentum) have become a stronger signal as the data improved statistically and systematically.

On the assumption that others kinds of anomalous events might occur, perhaps at new machines later this decade, I will divide this talk into two parts. First, I will consider some of the kinds of constraints that must apply to any attempts to understand the origins of anomalous events, with an emphasis on high energy colliders. The first requirement on a theory is obviously that some events of the "observed" kind are produced, i.e., that a specific mechanism exists. Equally important is that the rate of production of such events is reasonable and that other associated (but not observed) events are not produced. Most published models do not do well in this regard. It is very instructive to see the kinds of things that go wrong; today's unobserved prediction may be tomorrow's signal.

Then I will concentrate on the predictions of supersymmetry ${ }^{4}$ and whether it can describe the monojet events. As first emphasized in references 5 and 6 and by F. Paige

\footnotetext{
${ }^{a}$ This research was supported in part by the U.S. Department of Energy.
} 
(unpublished), extensive further analysis is necessary to compare theoretical predictions with data under actual experimental conditions; this is described fully in the following. The calculations I present are from an extensive collaboration ${ }^{7}$ with $M$. Barnett and H. Haber. Supersymmetry is a suprisingly well constrained theory in terms of comparison with experiment. Only the masses of the superpartners are unknown; once they are specified, all cross sections and distributions are fixed. Some kinds of events must occur (such as one-sided events with one or two jets) and others must not occur (e.g., any event with no missing momentum or any event with a hard $\gamma$ ). It is by no means trivial to find events of the right kind also occurring at the right rate and with the right distributions in jet momentum and in missing momentum. As we will see, at the present time there is (at least) one choice of masses that appears to be consistent with the monojet data and with other information concerning anomalous events.

It is very interesting that of the many types of anomalous events for which there were candidates during 1984, only the events with missing momentum (that arise naturally if supersymmetric partners are being produced) still seem to exist in the 1985 data.

\section{POSSIBLE SIGNATURES OF ANOMALOUS EVENTS}

There are a number of categories of events where anomalous effects might stand out. It may be useful to list some of these. Normally, if one kind of event occurs in a model, so do others.

(A) Signatures with missing transverse momentum $\left(p_{T}\right)$ :

\begin{tabular}{|c|c|}
\hline Topology & SM Background \\
\hline$j \not p_{T}$ & $\begin{aligned} W \rightarrow \tau \nu_{x}, g+Z, & \text { QCD } \\
& \left.\left.\right|_{\rightarrow \text { hadrons }}\right|_{\rightarrow, v}\end{aligned}$ \\
\hline$j j \phi_{T}$ & $g+\underset{\left.\right|_{\rightarrow \vec{y}}}{W}$ \\
\hline$\ell^{ \pm} p_{T}$ & $W \rightarrow \ell \nu$ \\
\hline$\ell^{ \pm} j \not \phi_{T}$ & $g+\underset{\left.\right|_{\rightarrow \ell v}}{W}$ \\
\hline$\ell^{+} \ell^{-} \not \phi_{T}$ & $Z \rightarrow \tau^{+} \tau^{-}$ \\
\hline
\end{tabular}

In all cases, the background can be measured experimentally when sufficient data is available and it can be quite accurately calculated as well. All of the above signatures are predicted to occur, in varying amounts, in supersymmetric theories.

(B) Signatures involving peaks:

\begin{tabular}{|c|c|}
\hline Topology & SM Background \\
\hline$\ell^{+} \ell^{-}$ & toponium, $\eta_{t}$ \\
\hline$j j$ & $\eta_{t}$ \\
\hline$j j j$ & $W+j, Z+j+$ cuts and phase-space \\
\hline$j W$ & $W+j, Z+j+$ cuts and phase-space \\
\hline$j Z$ & $W+j, Z+j+$ cuts and phase-space \\
\hline
\end{tabular}


(C) Signatures involving hard $\gamma$ 's:

\begin{tabular}{lll}
\cline { 1 - 1 } \multicolumn{1}{c}{ Topology } & & SM Background \\
\cline { 1 - 1 }$\gamma \gamma$ & & $\eta_{t}$, QCD \\
$\ell^{+} \ell^{-} \gamma$ & & bremsstrahlung \\
$\gamma \not_{T}$ & & $\gamma Z$ \\
$\gamma j$ & & QCD \\
$\gamma \nu \bar{\nu}$ & & $\gamma Z$ \\
$\ell^{ \pm} \gamma \phi_{T}$ & & \\
$\gamma \gamma \not_{T}$ & & \\
$\gamma Z$ & & QCD \\
$\gamma W$ & QCD
\end{tabular}

(D) Other constraints that are easily gotten wrong can be listed:

(1) The number of events has to be right, i.e., $\sigma \times \mathrm{BR}$, where $\sigma$ is the production cross section for the mechanism in question and BR is the branching ratio for the decays that lead to the signature in question. Many proposals give wrong rates; we will return to this below.

For explanations involving production of a single particle, there are "unitarity" constraints that exclude some (proposed) interpretations; they are not rigorous "unitarity" constraints since they require adding a physical condition that necessitates that the production partial width is smaller than the available energy region, but they give important constraints in practice. ${ }^{8,9}$

The basis is that for production of a single particle, $X$, of arbitrary spin and color from constituents, $a / b$, one has

$$
\left.\frac{d \sigma}{d y}\right)_{y=0}(\overline{\mathrm{p}} \mathrm{p} \rightarrow X+----)=\frac{2 J_{X}+1}{\left(2 S_{a}+1\right)\left(2 S_{b}+1\right)} \frac{N_{c}^{X}}{N_{c}^{a} N_{c}^{b}} \frac{\Gamma(X \rightarrow a b)}{M_{X}^{3}} \Lambda,
$$

where $N_{c}$ is the color multiplicity $(1,3,6,8,--)$ and $\Lambda$ depends on the structure functions,

$$
\Lambda=16 \pi^{2}\left[F_{a / \mathrm{p}}(x) F_{b / \bar{p}}^{\prime}(x)+(\mathrm{p} \leftrightarrow \overline{\mathrm{p}})\right] .
$$

Clearly, if $\Gamma(X \rightarrow a b)$ is limited, either by outside information or by the observation that (say) all events are in a region of $40 \mathrm{GeV}$ (or whatever value is appropriate), the possible rate is limited. At $M_{X} \simeq 100 \mathrm{GeV}$, the constraint is important and for $M_{X} \geq 150 \mathrm{GeV}$, it is very serious.

Barger et al. ${ }^{8}$ integrate over $y$ and define

$$
\sigma(\mathrm{p} \overline{\mathrm{p}} \rightarrow X+--)=\left(2 J_{X}+1\right) N_{c}^{X} \sum_{a b} \Gamma(X \rightarrow a b) R_{a b},
$$

thus giving useful graphs of $R_{a b}$ versus $M_{X}$ at various energies. Dusedau et al. ${ }^{9}$ study the constraints in detail for scalar particles.

Note that what is actually observed is

$$
\sigma \times \mathrm{BR}(X \rightarrow f)=\Lambda^{\prime} \frac{\Gamma}{M_{X}^{3}} \mathrm{BR}(X \rightarrow a b) \mathrm{BR}(X \rightarrow f),
$$


where $f$ is the final state of interest. Taking $\Gamma(X \rightarrow a b) \leq 50 \mathrm{GeV}$ (assuming the events show up in a limited energy region) and

$$
\mathrm{BR}(X \rightarrow a b) \mathrm{BR}(X \rightarrow f) \leq 1 / 4,
$$

there is an absolute constraint. Often, of course, the product of branching ratios is much less than $1 / 4$.

In general, it is possible to conclude that to explain anomalous events with single particle production, one must have $2 J_{X}+1$ and/or $N_{c}^{X}$ greater than unity and that major branching ratios must be involved.

(2) If a $Z^{0}$ is involved in the mechanism, a number of constraints operate:

(i) if a large branching ratio is involved, it can increase $\Gamma_{z}$;

(ii) probably, rates are large enough to see ${ }^{10}$ any anomalous $Z^{0}$ decay at PETRA, or PEP as well, via $e^{+} e^{-} \rightarrow Z \rightarrow$ ?

(iii) if a $Z^{0}$ mode is the origin of anomalous decay, other $Z^{0}$ modes must appear; e.g., if $\ell^{+} \ell^{-} \gamma$ events were supposed to be $Z^{0} \gamma$ with $Z^{0} \rightarrow \ell^{+} \ell^{-}$, then $\gamma \nu \bar{\nu}$ events must appear with $\sigma(\gamma \nu \bar{\nu}) / \sigma\left(\gamma e^{+} e^{-}\right)=$ 6 , and $\gamma j j$ events must appear with a known, ${ }^{11}$ large branching ratio before corrections for soft and coalesced jets.

(3) A number of distributions are characteristic of specific mechanisms. One can plot $\mathrm{p}_{T}^{j}, \phi_{T}, \theta\left(\ell^{ \pm} \gamma\right), M_{T}\left(j p_{T}\right)$, etc. We will see below that the distributions can be as powerful as one might expect in distinguishing competing mechanisms.

(4) The characteristics of jets are very important. They are also very sensitive to cuts. Typical jet charged particle multiplicities in the momentum range in question are large, with $n_{c h} \sim 10-20$. However, most of these particles are soft. If a cut is made to take only particles with $\mathrm{p}_{T}>1 \mathrm{GeV}$, then $n_{c h}$ drops greatly for quark jets, with $50-90 \%$ of the particles being softer than $1 \mathrm{GeV}$. If, as sometimes happens, a jet is a combination of two jets coalesced, the charge multiplicity will be double. Similar effects happen to jet effective masses.

The UA1 monojets have rather low mass and charged multiplicity. It seems to be very unlikely that they could be pairs of particles such as $q \bar{q}, q g, g g$. Whether a single $q$ or $g$ can fragment into that few particles $\left(n_{c h} \leq 4\right.$ for most monojets) is not yet clear, but it seems reasonable. Paige ${ }^{12}$ has examined this question with the ISAJET Monte Carlo, which gives a satisfactory description of jet multiplicities and masses at $e^{+} e^{-}$colliders. When he produces events with hard quark jets and UAl cuts, he finds the mean $n_{c h}$ is 4.2 , with $39 \%$ having $n_{c h} \leq 3$. However, when he examines a jet where $\tilde{g} \rightarrow q \bar{q} \tilde{\gamma}$ and $q \vec{q}$ coalesce, he finds, as expected, about double the multiplicity.

Probably, it is fair to conclude at present that the "narrow" jets observed can originate with single hard quarks and perhaps single gluons; however, any mechanism where they originate with coalesced jets must provide an explanation of the jet properties in order to be taken seriously. Further, if the jets originate as single quarks, some less narrow ones must appear, so the mean $n_{c h} \simeq 4$.

Many examples could be given of models that can provide one type of anomalous 
event, but that all too of ten provide too many of another. A few illustrations are:

(i) Any model based on heavy quarkonium states (formed from known or hypothetical new quarks) that give decays with $\ell^{+} \ell^{-} \gamma$ or decays with missing momentum produces ${ }^{13}$ a large number of $\ell^{+} \ell^{-}$ events at the quarkonium mass. This occurs since the direct annihilation occurs: $Q \bar{Q} \rightarrow \gamma$ or $Z^{0} \rightarrow \ell^{+} \ell^{-}$. No energetic $\ell^{+} \ell^{-}$ pairs are observed, excluding all such models.

(ii) Most models that produce a $Z^{0}$ in the decay of a heavy object also produce decays with the $Z^{0}$ replaced by a $W^{ \pm}$and by a $\gamma$. The absence of many candidates for events with hard $\gamma$ 's is a severe constraint on models.

(iii) There is an event reported by the CELLO detector at DESY, at 44 $\mathrm{GeV}$ near the top machine energy, of $j j \mu^{+} \mu^{-}$. The four particles are in one plane, which is very unlikely unless the event is due to a pair of heavy particles produced essentially at rest. However, if the particles are leptoquark states, $L Q \rightarrow j \mu$, the cross section to produce them at CERN is large (they would be like $20 \mathrm{GeV}$ $t$-quarks) and their signature is very clear, so this is excluded. On the other hand, though, they could be heavy neutrinos, with $\nu^{\prime} \rightarrow \mu q \bar{q}$, but then the jet would be a coalesced $(q \bar{q})$ one of large effective mass and multiplicity, while the experimental one is a typical light quark jet. Therefore, no interpretation is consistent.

We will see more examples of constraints below.

\section{CATEGORIES OF INTERPRETATIONS}

The interpretations of anomalous events can be put into four main categories:

(1) New $Z^{0}$ decay modes;

(2) Decays of a singly produced new particle;

(3) Associated production of new particles;

(4) Profound.

The last category, of which examples are given in references 15 and 16 , argues that a new interaction may be showing up, but not in a simple way, via new low-spin particles. Supersymmetry either combines categories (2) plus (3) or just uses category (3), depending on the masses involved.

\section{APPROACHES TO MONOJET EVENTS}

Since currently the monojet events are the main candidate for a signal for anomalous events, I will comment on various approaches to their interpretation. First, I will describe three approaches that-if the data is taken at face value-do not easily 
give an acceptable interpretation, but involve interesting and instructive physics. Then I will turn to the supersymmetric approach and describe how some choice of masses may successfully describe the present monojet data.

On the basis of the comments in the previous pages and from the reported monojet (and dijet) data, I will require any explanation to have as necessary conditions:

(i) about the right number of events, including effects of experimental cuts, detection efficiencies, etc.;

(ii) some one-sided dijets (even though the experimental situation and the comparison of experiment and theory is not straightforward here-see below);

(iii) most jets must be due to a single quark and probably not to coalesced jets;

(iv) the monojet distributions, especially the $M_{T}\left(j p_{T}\right)$ distribution, must look like the data;

(v) no other wrong predictions.

\section{CAN $Z^{0}$ DECAYS EXPLAIN THE MONOJETS?}

Two interesting approaches to monojets via $Z^{0}$ decays are (a) that of Krauss, ${ }^{17}$ followed by references 18 and 19 , where $Z^{0} \rightarrow \nu^{\prime} \nu^{\prime \prime}$, and where the objects, $\nu^{\prime}$ and $\nu^{\prime \prime}$, are new or familiar neutrino-like particles, which may either escape the detector providing $p_{T}$, or decay, providing narrow hadronic jets; and (b) that of Glashow and Manohar, ${ }^{10}$ Moffat, ${ }^{20}$ and King, ${ }^{20}$ where $Z^{0} \rightarrow H_{1}^{0} H_{2}^{0}$, with $H_{3}^{0}$ and $H_{2}^{0}$ being two light neutral scalar or pseudoscalar (perhaps Higgs) bosons that arise in models with two doublets or with extra gauge groups. One $H_{1}^{0}$ is constrained to be effectively invisible, either by requiring it to be very light so only $\gamma \gamma$ and $e^{+} e^{-}$modes are allowed or by largely decoupling it. The other $H_{I}^{0}$ is light enough to appear as a hadronic jet when it decays.

These approaches do not seem to me to give likely explanations of the data, for reasons I list below, but they have some nice points and should be kept in mind as the data improves. Among their virtues are that they can easily give the right number of events since the associated $Z^{o}$ branching ratios are in the $1-3 \%$ range, which is about what is needed, and that the events would be centered near $M_{Z}$ in the $M_{T}$ distribution. As the data increases, the latter point seems to be less of a virtue because the data does not peak in $M_{T}$ and it spreads out over $80 \mathrm{GeV} \leq M_{T} \leq 120 \mathrm{GeV}$.

Their problems are:

(1) The experimental $M_{T}$ distribution is too broad and not sharply peaked;

(2) For $H_{1}^{0}$ giving the monojet, it seems very hard to get $n_{c h} \leq 4$ for most events. The $H_{I}^{0}$ will probably have to decay to $\bar{c}$, which will give $n_{c h} \geq 5$ for most events;

(3) For $\nu^{\prime}$ giving the monojet, one always needs to have a charged lepton in the jet (since $\nu^{\prime} \rightarrow \ell \pm q \bar{q}$ ), but almost none of the monojets are reported to contain a hard charged lepton;

(4) No primary dijet events are generated by this mechanism, so the dijets must come from the primary production of $g Z^{0}$ and are suppressed in rate. 


\section{CAN TECHNICOLOR EXPLAIN THE MONOJETS?}

Technicolor was and is a good idea, ${ }^{21}$ that the new physics required by spontaneous symmetry breaking is due to the dynamics of some new fermions. Some bound states also play the role of Higgs bosons. Then, normally, other new particles arise and give a number of interesting signatures. Some of the particles, their masses, and their decays are listed in TABLE 1 ( $N$ is a group label, with $N \geq 4$ expected). Although the simplest masses, with $N=4$, are not appropriate for the current CERN data, perhaps $N$ is larger or maybe a different symmetry gives lower masses; or perhaps such signatures will appear at a future collider. From the table,$^{21}$ we can immediately see both some interesting signatures, such as $t \bar{t}$, busy $Z$ 's or busy $W^{ \pm}$( $Z$ 's or $W$ 's accompanied by jets), and $g \gamma$ events. The $g \gamma$ events would be very dramatic, appearing as $j \gamma$, and would have a large cross section. Monojet events would appear as $g Z^{0}$ with $Z \rightarrow \nu \bar{\nu}$ and would then require events with $j \ell^{+} \ell^{-}, j \gamma$, and $j j j$, all of which are not seen. Pair production of leptoquarks $(L Q)$ would be important if $M(L Q) / M\left(\eta_{T}\right.$ or $\left.\pi_{T}\right) \simeq 2 / 3$ was maintained, again giving interesting signatures. Altogether, it is clear that technicolor signatures are not what is being observed, but it will be interesting to look again in the future. ${ }^{22}$

TABle 1. Possible Anomalous Events from Technicolor

\begin{tabular}{|c|c|c|c|}
\hline Particle & Mass (GeV) & Color & Decays \\
\hline \multicolumn{4}{|c|}{ Singly Produced } \\
\hline$\eta_{T}$ & $\sim 240 \sqrt{4 / N}$ & 8 & $\begin{array}{l}\bar{t} \overline{90 \%} \\
g g 5 \% \\
g Z^{0} 10 \%\end{array}$ \\
\hline $\boldsymbol{\pi}_{T}^{ \pm}$ & $\sim 240 \sqrt{4 / N}$ & 8 & $\begin{array}{l}t \bar{b} 75 \% \\
g W^{ \pm} 20 \%\end{array}$ \\
\hline$\pi_{T}^{0}$ & $\sim 240 \sqrt{4 / N}$ & 8 & $\begin{array}{l}g Z^{0} 30 \% \\
g \gamma 70 \%\end{array}$ \\
\hline \multicolumn{4}{|c|}{ Pair Produced } \\
\hline$L Q$ & $\sim 160 \sqrt{4 / N}$ & 3 & $\begin{array}{l}t \tau^{+}, t \bar{v}, b \tau^{+}, b \bar{\nu} \\
j \mu, j e, j v\end{array}$ \\
\hline
\end{tabular}

\section{CAN $W W$ "BOUND STATES" GIVE MONOJETS?}

Although proponents of complicated dynamics ${ }^{15,16}$ have not proposed specific models for monojets, one can examine a few alternatives to see if any simple interpretation arises. Although none has so far, this does not necessarily imply that the approach is wrong; only that more subtle thinking would be needed if such approaches were relevant. The following remarks are my own approach to such questions and do not reflect the views of other authors.

Two general problems with such approaches will be (i) that the decay $q p_{T}$ of a color-singlet $W W$ bound state is not possible, so it will be hard to get narrow, low multiplicity jets plus missing momentum, and (ii) one might expect a single, rather 
narrow state so far below threshold, which would give problems with the $M_{T}$ distribution.

One way to get narrow jets would be a vector dominance mechanism, with one $W \rightarrow \pi, \rho$. Then monojets could arise from a $W^{ \pm} Z^{0}$ state with

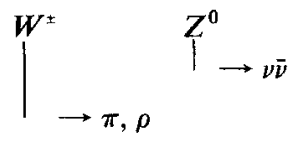

or a $Z^{0} Z^{0}$ state with

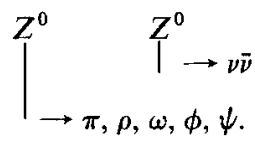

The main problems with this mechanism are rate/branching ratio and other modes. In particular, if this occurred, one would also expect

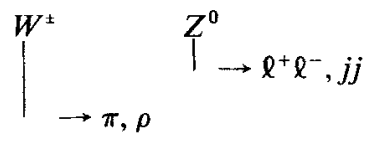

and

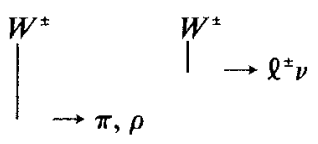

giving large numbers of $\ell^{ \pm} j \not p_{T}$ events, which may not be observed. (However, note the UA2 events.)

\section{CAN SUPERSYMMETRY EXPLAIN MONOJET EVENTS?}

We have seen that no proposed approach we considered above seems to give a clean explanation of the monojet events. It has been known for a long time ${ }^{23}$ that events with jets and missing momentum would occur if supersymmetric partners were being produced. Now, we will consider whether the detailed characteristics of supersymmetric particle production are consistent with the monojet data. All the work I describe in this section is based on a collaboration ${ }^{7}$ with M. Barnett and H. Haber, plus the work of other authors. We will see that there is indeed a satisfactory description, perhaps even competing ones, as far as the monojet data is concerned. Unbalanced dijet events are also predicted and some are found. The detailed properties of the predicted ones and the observed ones are not quite the same, however, and whether this is a problem is not yet clear.

The minimal spectrum of new particles expected if supersymmetry were a 
symmetry of nature as realized on the weak scale is that each of the quarks, leptons, and gauge and Higgs bosons should have a partner whose properties are identical to the particle except that it differs in spin by half a unit. A useful notation and nomenclature is indicated in TABLE 2. The names indicate both the origin of the particle and, for the partners of gauge bosons and Higgs bosons, how the partner interacts; because of weak interaction eigenstates mixing to form mass eigenstates, the interaction properties can be subtle, but they are important to determine observability. Left-(L) and righthanded( $R$ ) fermions are separated since they have different weak interactions.

If supersymmetry were an unbroken symmetry, the partners would have the same mass, color, charge, etc., as the particles, and they would have been easily detected. Since they have not been, we consider them to be heavier because of symmetry breaking. If supersymmetry is relevant to explaining the weak scale, presumably it makes sense to expect the partners to have mass, $\sim M_{W}$. If partners are not discovered

Table 2. The Minimal Particle Spectrum in a Supersymmetric Theory

\begin{tabular}{|c|c|c|}
\hline Normal Particles & Superpartners & Names \\
\hline$g$ & $\tilde{g}$ & \multirow{2}{*}{$\begin{array}{l}\text { gluino } \\
\text { up-squark }\end{array}$} \\
\hline$u_{L}$ & $\tilde{u}_{L}$ & \\
\hline$u_{R}$ & $\tilde{u}_{R}$ & $\cdot$ \\
\hline . & $\cdot$ & $\cdot$ \\
\hline . & . & \multirow{2}{*}{ selectron } \\
\hline$e_{L}$ & $\tilde{e}_{L}$ & \\
\hline$\cdot$ & $\cdot$ & \multirow{2}{*}{$\cdot$} \\
\hline . & & \\
\hline$W^{ \pm}$ & $\tilde{W}^{ \pm}$ & \multirow{2}{*}{$\begin{array}{l}\text { wino } \\
\text { whiggsino } \\
\text { higgsino }\end{array}$} \\
\hline & & \\
\hline$H_{l, 2}^{ \pm}$ & $\tilde{h}_{1,2}^{ \pm}$ & higgsino \\
\hline$\gamma$ & $\tilde{\gamma}$ & \multirow{2}{*}{$\left.\begin{array}{l}\text { photino } \\
\text { zino } \\
\text { higgsino }\end{array}\right\}$} \\
\hline$Z$ & $\tilde{Z}$ & \\
\hline$H_{1,2}^{0}$ & $\tilde{h}_{1,2}^{0}$ & neutralinos \\
\hline
\end{tabular}

on the weak scale, eventually the limits on their masses will be pushed up to well beyond the weak scale and they will become irrelevant to the understanding of physics on the weak scale.

Since the way in which the supersymmetry is broken is not understood, the masses of the superpartners are unknown. It is necessary to proceed by assuming a set of masses (in practice that means masses for mainly $\tilde{g}, \tilde{q}, \tilde{w}, \tilde{\ell} \tilde{l}^{ \pm}, \tilde{\nu}$, and $\tilde{\gamma}$ ), calculating predictions, and testing them.

Fortunately, the couplings of the superpartners are known because they are mainly gauge couplings. In fact, they are the actual measured gauge couplings, so no uncertainty arises at all. The rule that emerges from the theory is that every vertex in the standard theory has added other vertices obtained by replacing particles by their partners in pairs, while keeping the interaction strength the same. Thus, the $\bar{q} q g$ coupling gives $\tilde{q} \tilde{g} q$ and $\tilde{q} \tilde{q} g$ couplings, all of strength, $g_{3}\left(g_{3}^{2} / 4 \pi=\alpha_{s}\right)$, the Wev coupling gives $\tilde{w} \tilde{e} \nu, \tilde{w} e \tilde{v}$, and $W \tilde{e} \tilde{v}$ couplings, all of strength, $g_{2}$, etc. With this rule, one can draw 
all the Feynman graphs that are relevant to whatever process is being considered and estimate any cross section or decay.

Since supersymmetric partners occur in pairs, they can either be pair produced or a single one can be produced via $\tilde{q}$ or $\tilde{g}$ in a proton. ${ }^{24,25}$ (The other supersymmetric particle goes down the beam pipe with other soft partners.) We will consider both mechanisms; it turns out that if gluinos are lighter than about $25 \mathrm{GeV}$, the gluino content of a proton is significant.

Once a superpartner is produced, it will decay into normal particles plus a lighter superpartner; the lightest superpartner will be stable (one can consider modified cases where the lightest superpartner is unstable and carry through the analysis in a way analogous to the following, but little changes and we will not pursue this alternative here). The lightest superpartner can be a partner of a gauge boson or a Higgs boson, or it can be a sneutrino. For our purposes, it will not matter much what is the lightest superpartner, just as long as its essential property is that it normally escapes collider detectors. This is so because to interact, it must excite a superpartner in the detector, and since the partners of quarks and leptons are heavy, the interaction cross section is at most of order, $\alpha^{2} / \tilde{m}^{2}$, with $\tilde{m} \gtrsim 20 \mathrm{GeV}$, which gives too small a cross section to see.

Thus, the basic signature of the production of supersymmetric partners is missing momentum, accompanied by jets or charged leptons in characteristic patterns. If a squark is produced, it will decay into $q \tilde{g}$ and $q \tilde{\gamma}$ if both are kinematically allowed, in a ratio (ignoring phase-space) of

$$
\frac{\Gamma(\tilde{q} \rightarrow q \tilde{\gamma})}{\Gamma(\tilde{q} \rightarrow q \tilde{g})}=\frac{3 e_{q}^{2} \alpha}{4 \alpha_{s}} .
$$

For $\tilde{u}$, this is $\alpha / 3 \alpha_{s} \approx 0.02$. If $\tilde{m}_{q} \gg \tilde{m}_{w}, M_{q}$, then

$$
\frac{\Gamma(\tilde{q} \rightarrow q \tilde{w})}{\Gamma(\tilde{q} \rightarrow \tilde{q} g)} \simeq \frac{1}{2} \frac{3 \alpha_{2}}{8 \alpha_{\mathrm{s}}} \simeq 0.05,
$$

where the $1 / 2$ appears because only $\tilde{q}_{L}$ has a coupling to $\tilde{w}$. Similarly, if $\tilde{\ell}^{ \pm}$appear, they will decay as $\tilde{\ell}^{ \pm} \rightarrow \ell^{ \pm} \tilde{\gamma}$. Gluinos will mainly decay via a virtual $\tilde{q}, \tilde{g} \rightarrow \bar{q} q \tilde{\gamma}$, with a branching ratio (that depends on other masses) of perhaps $1 \%$ to $g \tilde{\gamma}$.

If sleptons or sneutrinos are lighter than winos or zinos, the latter will have useful signatures,

$$
\begin{gathered}
\tilde{w} \rightarrow \tilde{\ell}^{ \pm} \nu \rightarrow \ell^{ \pm} \not_{T}, \\
\tilde{w} \rightarrow \ell^{ \pm} \tilde{\nu},
\end{gathered}
$$

or

$$
\tilde{z} \rightarrow \tilde{\ell}^{+} \ell^{-} \rightarrow \ell^{+} \ell^{-} \not p_{T} .
$$

If sleptons and sneutrinos and also $\tilde{g}$ are heavier than winos and zinos, the latter still may have a useful signature,

$$
\begin{gathered}
\tilde{w} \rightarrow \bar{q} q \tilde{\gamma} \rightarrow j j \phi_{T}, \\
\tilde{z} \rightarrow \bar{q} q \tilde{\gamma} \rightarrow j j \phi_{T} .
\end{gathered}
$$


If only $g$ are lighter than $\tilde{w}, \tilde{z}$, the latter will decay into $j j j j \tilde{\gamma}$, becoming essentially unobservable. In the approach we favor, with $\tilde{M}_{g} \simeq 10 \mathrm{GeV}$, this is perhaps the most likely situation, though it could well happen that sleptons or sneutrinos are lighter than $\tilde{w}$ or $\tilde{z}^{26}$

\section{PRODUCTION OF SUPERPARTNERS}

There are two kinds of ways to produce superpartners at a hadron collider.

(A) Starting with quarks and gluons in the proton, we have ${ }^{27}$

$$
\sigma(\mathrm{p} \overline{\mathrm{p}} \rightarrow \tilde{a} \tilde{b} X)=\int d x_{1} d x_{2} F_{c}\left(x_{l}\right) F_{\bar{d}}\left(x_{2}\right) \hat{\sigma}(c \bar{d} \rightarrow \tilde{a} \tilde{b}),
$$
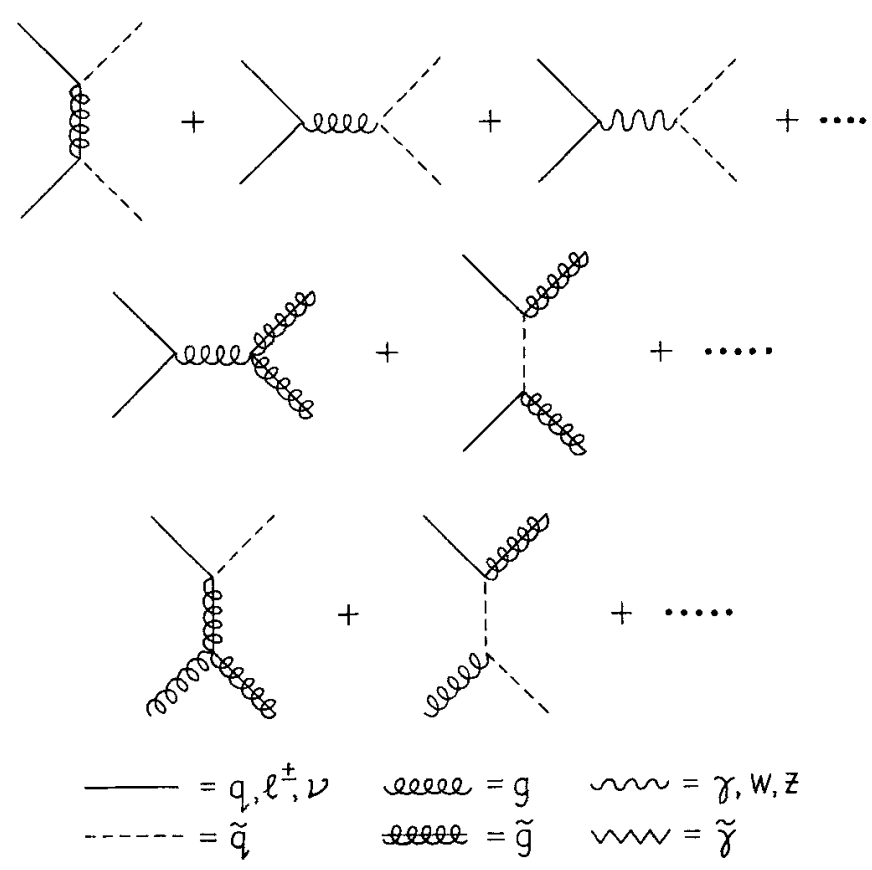

FIGURE 1. Some ways to produce superpartners. At $\tilde{q} \tilde{q}$ vertices, $\tilde{g}$ can be replaced by $\tilde{w}, \tilde{z}$, or $\tilde{\gamma}$, giving production of $\tilde{w} \tilde{w}, \tilde{w} \tilde{g}, \tilde{g} \tilde{\gamma}, \tilde{z} \tilde{g}$, etc.

where $F_{c}$ is the structure function for constituent $c$ in a proton, $F_{d}$ is the structure function for constituent $d$ in a proton, and $\hat{\sigma}$ is the constituent cross section. FIGURE 1 shows a number of constituent processes to produce superpartners; which ones dominate will depend on masses, kinematic regions, cuts, etc. In all cases, we include all subprocesses in our calculations. We have calculated all cross sections to produce $\tilde{q} \tilde{q}, \tilde{g} \tilde{g}, \tilde{g} \tilde{q}, \tilde{w} \tilde{g}, \tilde{g} \tilde{\gamma}$, etc., and comparted with earlier results in reference 27 . We use the EHLQ structure functions ${ }^{28}$ 
for calculations, with $\Lambda=0.29 \mathrm{GeV}$. Then, events are generated by a Monte Carlo integration technique ${ }^{29}$ and final state particles are decayed, using a procedure due to two of us. ${ }^{29}$ (Note that we have not included any effects of QCD gluon radiation or " $K$-factors" in the calculations.)

Ultimately, an important test of whether supersymmetric partners are being produced will be the determination of the spins of the new particles. While it is clearly too early to be doing that, the spins do affect the distributions and rates we predict, so their effects are being included. At the present level of calculation, no polarization effects are expected; transverse polarizations are absent since we are assuming parity conservation. Supersymmetric processes in general do not conserve parity since masses of $\tilde{q}_{\mathrm{L}}$ and $\tilde{q}_{\mathrm{R}}$ are not in general equal. By including such mass differences, longitudinal

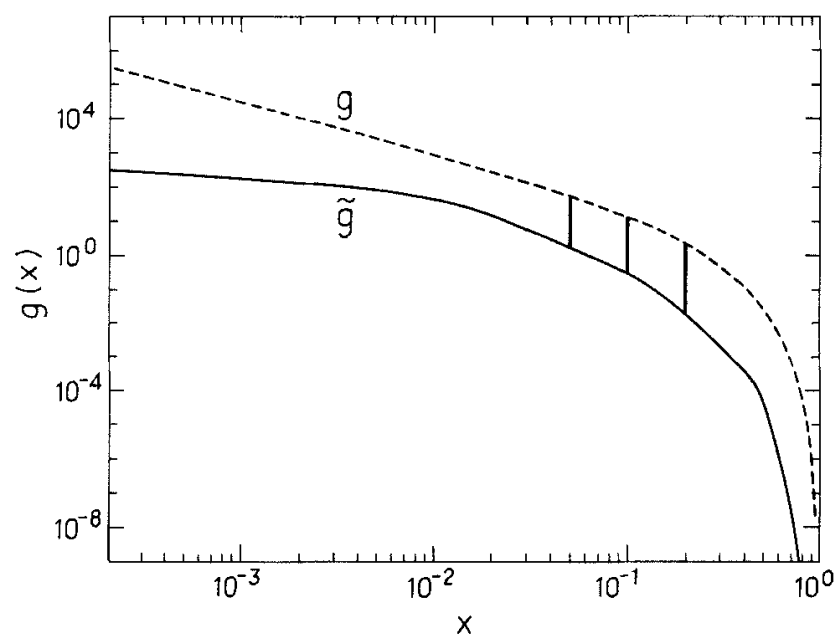

FIGURE 2. Gluino distribution function, from reference 7.

polarizations would be generated, but at the present time such effects are highly model dependent and we will neglect them.

(B) If colored superpartners were light, they would occur ${ }^{24,25}$ in hadrons as well as normal colored quarks and gluons. Since squarks are known to be heavier than about $20 \mathrm{GeV}$, they will not appear in significant quantities in a proton. The probability of a gluon splitting into a gluino pair is six times larger than the probability of it splitting into a quark pair of the same mass, so even if $\tilde{M}_{g}$ is $20-25 \mathrm{GeV}$, there is a significant ${ }^{7}$ gluino content to the proton.

The question of gluinos in a proton has been raised in reference 24 , and Herrano et $a .^{25}$ have specifically suggested that single squark production would occur via this mechanism. We have calculated the gluino structure functions, including mass effects, ${ }^{7}$ by using the technique proposed by Gluck, 
Hoffman, and Reya (based in turn on the Altarelli, Parisi equations) and by checking against the EHLQ calculations of the heavy quark content of a proton. Since $\tilde{m}_{g}^{2} / Q^{2} \leqslant 1 / 16$ and even though there are corrections of the order, $\tilde{M}_{g}^{2} / Q^{2}$ and $\left(\tilde{M}_{g}^{2} / Q^{2}\right)^{2}$, involved, the lowest order ones, which are the most likely to be correct, are dominant. FIGURE 2 shows the gluino structure functions obtained. The curve for $d \sigma / d x$ peaks around $x \simeq 0.08$, where the calculation is expected to be reliable, with $x$ neither large nor small when compared to $\tilde{M}_{g}^{2} / Q^{2}$ for the values of $\tilde{M}_{g}^{2}$ we find to be relevant.

\section{EXPERIMENTAL CUTS}

In a real experimental environment, it is necessary to apply cuts to the data ${ }^{30}$ in order to exclude conventional sources of anomalous events. The most important cut is on the minimum $\phi_{T}$ required to count the event as a monojet event. Data has been presented $^{2}$ in various ways, with fixed cuts such as $\phi_{T}>40 \mathrm{GeV}$ and with a " $4 \sigma$ " cut. We will discuss how we implement these ${ }^{7}$ in the theory in the following section; it is subtle to make the " $4 \sigma$ " cut in a way where theory is being correctly compared to experiment. Other groups ${ }^{5,6,31-35}$ have already done similar analyses. It is a difficult procedure and not all analyses agree, though convergence is occurring.

In addition, an anomalous event must have one jet with $\mathrm{p}_{T}^{j}>25 \mathrm{GeV}$. Since $p_{T}$ and $\mathrm{p}_{T}^{f}$ are both large, clearly this biases all other activity in the event to be softer. A jet is defined ${ }^{2,30}$ as all activity in a bin, $\Delta \eta^{2}+\Delta \phi^{2} \leq 1$, where $\eta$ is the rapidity and $\phi$ is the azimuthal angle.

Some geometrical cuts are applied, as described in the UAl paper, ${ }^{30}$ and we have included these in our analysis.

\section{THEORETICAL CUTS}

In discussing the data, it is useful ${ }^{7}$ to consider three different cuts on $\not_{T}:$ (a) $\phi_{T}>40$ $\mathrm{GeV}$, (b) $\phi_{T}>32 \mathrm{GeV}$, and (c) $\phi_{T}>2.8 \sqrt{E_{T}}$ (i.e., the $4 \sigma$ cut). The first two are straightforward. The recently reported data is most complete for the $p_{T}>40 \mathrm{GeV}$ cut. The $\phi_{T}>32 \mathrm{GeV}$ results are presented as a comparison and as a prediction of how the number of monojet events changes as the cut is varied.

For the cuts on jet $\mathrm{p}_{T}$, we use the same values as the experimental analysis; $\mathrm{p}_{T}^{j}>12$ $\mathrm{GeV}$ for others. This is somewhat of a subtle point, as it is not clear whether a $25 \mathrm{GeV}$ parton corresponds to a $25 \mathrm{GeV}$ jet in the detector. Future analyses can improve on this.

The most subtle aspect of the analysis is defining the $4 \sigma$ cut. It is not necessary to consider this cut for the main part ${ }^{7}$ of our analysis (where we examine the $\not_{T}>40$ and $\not p_{T}>32$ distributions for all $\tilde{q}$ and $\tilde{g}$ masses), but it is necessary to compare with previous analyses, particularly of pair production of lighter gluinos, and it will be necessary for a full analysis in the future of all $p_{T}$ events due to both Standard Model and anomalous sources since they overlap near the $4 \sigma$ cut. Therefore, we go into some detail. ${ }^{7}$ 
The experimental missing energy fluctuations are characterized by a $\sigma=0.7 \sqrt{E_{T}}$, i.e., the amount of energy missing in an event goes as $\sqrt{E_{T}}$. To be safe in assigning an event to be new physics, it is required that $\phi_{T}$ be greater than $4 \sigma$. The problem is how to relate what happens in a theory event to $E_{T}$. The so-called "minimum bias" collider events (those with no large $\mathrm{p}_{T}$ activity) have a distribution in $E_{T}$ with a mean of $\left\langle E_{T}\right\rangle \simeq$ 20-25 GeV. Further, normal QCD jet-jet events are observed to have the property that when the two jets are removed, the remaining event has $\left\langle E_{r}\right\rangle \simeq 40-50 \mathrm{GeV}$.

Now suppose the monojets are due to the production of supersymmetric partners. Then presumably the total $E_{T}$ is given by

$$
E_{T}^{T o t}=E_{T}^{0}+E_{T}^{q, g},
$$

where $E_{T}^{0}$ is related to what is observed to QCD jet-jet events after the two jets are removed and where $E_{T}^{q .8}$ is the total energy associated with all of the partners that arise from the decay of supersymmetric particles, whether they pass the jet cuts or not. We take $E_{T}^{0}$ to have mean of $40 \mathrm{GeV}$ (using the lower side of the range because there is one less jet to radiate) and we use a distribution about $E_{r}^{0}$ to make the simulation as realistic as possible. ${ }^{7}$

This procedure gives a larger $E_{T}=E_{T}^{\text {Tot }}$ with which to calculate $\sigma$, so fewer events pass the cuts, and we do not find too many monojets arising from light gluinos that are pair produced. As a consequence, we do not find that any $\tilde{M}_{g}$ is clearly excluded by predicting significantly more monojet events than there are candidates for. In this connection, it should be noted that as the gluino mass decreases, it becomes less probable that a given $\tilde{g} \tilde{g}$ event passes the cuts, while the cross section for making the $\tilde{g} \tilde{g}$ pair grows. Since the probability of passing the cuts gets quite small $\left(\lesssim 10^{-3}\right.$ when $\tilde{M}_{g} \simeq$ $5 \mathrm{GeV}$ ), it is necessary to generate a large number of Monte Carlo events to have reliable results. While we have been careful to do this, we think further consideration of the low mass gluino pairs may be worthwhile.

\section{MONOJET EVENTS}

We have concluded that the regions of the $\tilde{M}_{\mathrm{g}}-\tilde{M}_{q}$ plane that could describe the data are those shown in Figure 3 . In the next sections, we study the allowed regions further. For monojets, ${ }^{b}$ one can get about the same conclusions from the distributions in $M_{T}\left(j, p_{r}\right), \mathrm{p}_{T}^{j}$ or $\phi_{T}$, so we mainly use $M_{T}$, which is easy to interpret physically. We find that, taking the data at face value, there seems to be a preference for the region with $\tilde{M}_{q} \simeq 110 \mathrm{GeV}$ and $\tilde{M}_{g} \simeq 10-20 \mathrm{GeV}$ where most of the monojets arise from single $\tilde{q}$ production via gluinos in the proton. While it is too soon to conclude that this is the only allowed region or that it is indeed the origin of the monojets, at present it may give a better description of the data than the other alternatives.

It is important to note that the reported monojet data has not been corrected for $\mathrm{p}_{T}^{j}$ mismeasurement. ${ }^{36}$ Indications from other jet information ${ }^{36}$ are that $\mathrm{p}_{T}^{j}, M_{T}$, etc., will

\footnotetext{
${ }^{b}$ Note that for dijet events, it is not so simple because of the extra jet. Our dijet predictions give a good description of the reported $M_{T}$ distribution, but not of the $p_{T}$ values.
} 


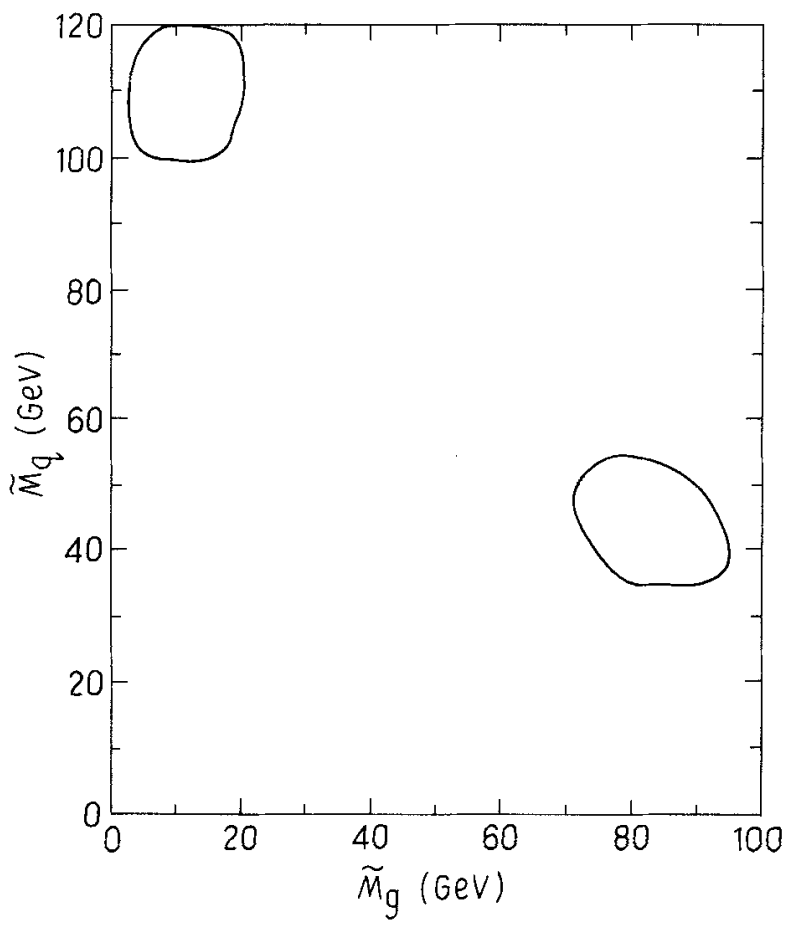

FIGURE 3. Allowed regions in the $\tilde{M}_{g^{-}} \tilde{M}_{q}$ plane for production of supersymmetric partners to explain monojet events, according to the criteria of reference 7 .

be corrected up by $5-10 \%$ eventually. We do not show such corrections, but they should be kept in mind.

\section{SINGLE SQUARK PRODUCTION}

We have explained above how the single squark is produced via the $\tilde{g}$ content of the proton. ${ }^{7}$ There are three sources of actual monojet events:

(1) $q \tilde{g} \rightarrow \tilde{q} \rightarrow q \tilde{g}$

$$
\stackrel{\mathrm{l}}{\rightarrow} \bar{q} \tilde{\gamma}
$$

This provides the majority of monojet events. The $q$ from $\tilde{q}$ decay is energetic and passes the jet cuts, and $5-10 \%$ of the time, the photino from $\tilde{g}$ decay passes the $\phi_{T}$ cut, giving an event that passes the UA1 cuts. There is, though, some residual energy from the two soft $q, \bar{q}$ from the $\tilde{g}$ decay.

(2) $q \tilde{g} \rightarrow \tilde{q} \rightarrow q \tilde{\gamma}$

This is about a $2 \%$ branching ratio, but it essentially always passes the cuts. It provides $10-15 \%$ of the monojet events. 
(3) Since gluinos are $10-20 \mathrm{GeV}$, their pair production cross section is large. As we discussed above, of the order $10^{-3}$ of the candidates pass the cuts. We find about $25-35 \%$ of the monojet events arising this way. Once $\tilde{M}_{g}^{2} \ll Q^{2} \simeq(100$ $\mathrm{GeV})^{2}$, the number of monojets arising from this mechanism is basically independent of $\tilde{M}_{g}$, as might be expected physically.

We note from the $M_{T}$ distribution with various cuts (FIGURE 4) that if the cut is placed at $p_{T}>32 \mathrm{GeV}$, almost as many new monojet events should occur as above $\hat{p}_{T}>$ $40 \mathrm{GeV}$. While not quite that many candidates are in the reported data, several candidates do exist, so at present this prediction is not inconsistent with the data. However, to accept the heavy squark interpretation, it will be crucial to see the additional events as the $p_{T}$ cut is reduced.

Note that there is some sensitivity to $\tilde{q}$ and $\tilde{g}$ masses, but not too much flexibility. The fraction of the events from $\tilde{q} \rightarrow q \tilde{\gamma}$ does shift as $\tilde{M}_{q}$ shifts, but because the other sources of events depend on the $\tilde{g}$ decay, they are not as sensitive. When high statistical data is available, an accurate determination of masses will be possible. If the $M_{T}$ distribution is shifted up 5-10\% from jet energy calibration, as expected, one can shift masses to compensate, but it is not a simple situation because cross sections will then decrease. If one takes the present numbers very seriously, though, $\tilde{M}_{g} \simeq 10-15 \mathrm{GeV}$ probably, but as the data improves this could change.

\section{SQUARK PAIR PRODUCTION}

The $M_{T}$ distribution for squark pair production ${ }^{37}$ is shown in FIGURE 5. If only the $p_{T}>40$ distribution were considered, this mechanism does as well as the single heavy

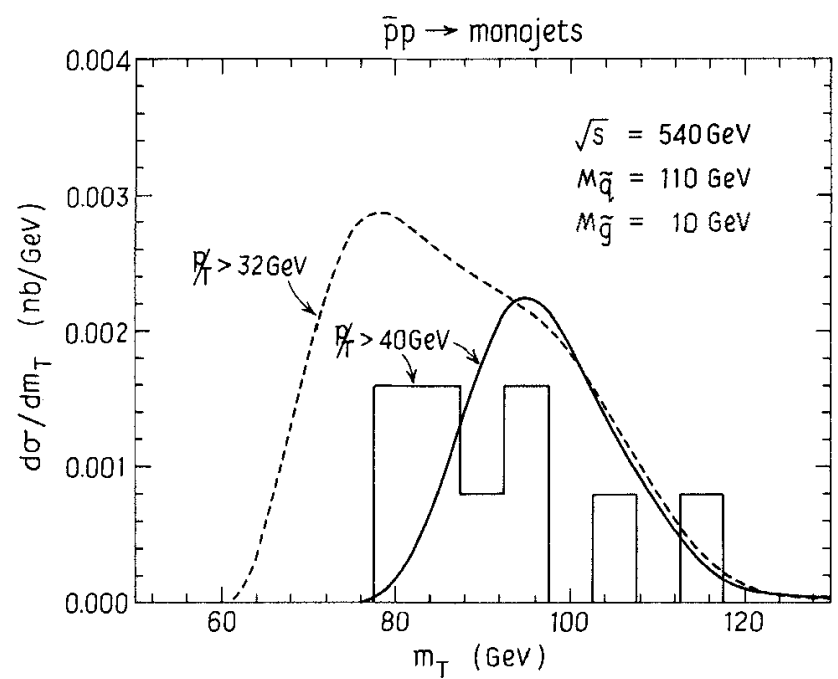

FIGURE 4. $M_{T}$ distribution for $\tilde{M}_{q}=110 \mathrm{GeV}, \tilde{M}_{g}=10 \mathrm{GeV}$, from reference 7 . Data from UAl, reference 3 . 


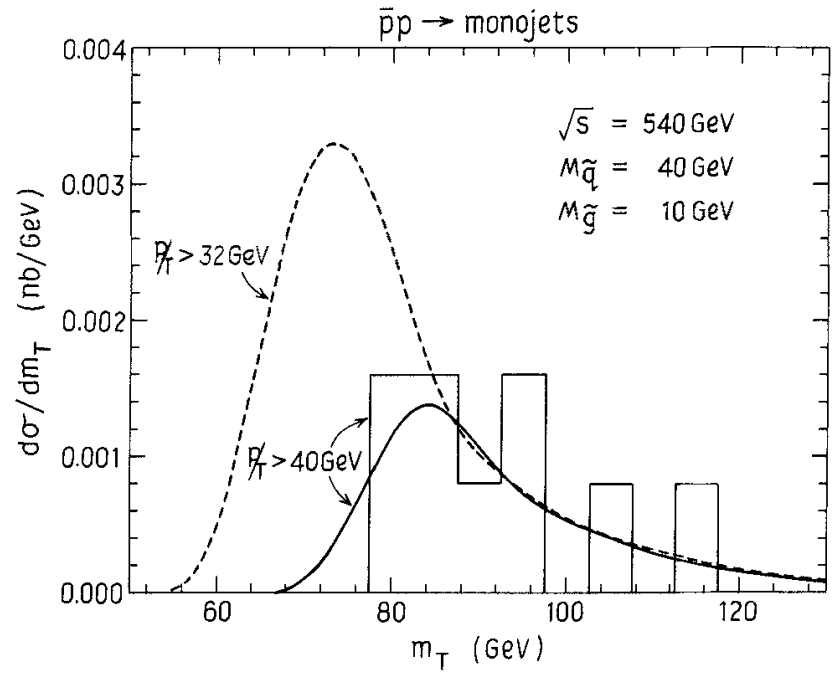

FIGURE 5. $M_{T}$ distribution for $\tilde{M}_{q}=40 \mathrm{GeV}, \tilde{M}_{g}=80 \mathrm{GeV}$, from reference 7. Data from UA1, reference 3 .

squark. However, the full $M_{T}$ distribution for $\tilde{q} \tilde{q}$ peaks strongly at $M_{T} \simeq 70-75 \mathrm{GeV}$, while the data is flat or a little peaked nearer to $90-100 \mathrm{GeV}$. If the $p_{T}$ cut is relaxed from 40 to $32 \mathrm{GeV}$, at least 1.5 times as many events should appear as were already found. However, the reported data contains only about two-thirds as many candidate events in that region as above $p_{r}=40 \mathrm{GeV}$. If one tries to shift the distribution up by increasing $\tilde{M}_{q}$, the cross section decreases. Therefore, if the data continues to be very flat or peak above $85-90 \mathrm{GeV}$, it will not be possible to obtain a consistent description of the data with $\tilde{q}$ pair production. The situation will get worse if the entire $M_{T}$ distribution is shifted up by $5-10 \mathrm{GeV}$ because of jet energy calibration. This is the basis of our preference for the single squark mechanism.

\section{HEAVY SQUARK AND VERY LIGHT GLUINOS}

Barger et al. have argued ${ }^{33,35}$ that an alternative mechanism, with a heavy $\tilde{q}\left(\tilde{M}_{g} \simeq\right.$ $3 \mathrm{GeV}$ ), gives a description of the data. While we find this is perhaps correct as to rate and perhaps as to the $M_{T}$ distribution, for two reasons we are concerned ${ }^{7}$ about the validity of this interpretation. Since the gluino in this scenario is either long-lived or fragments softly so that the resulting photino and jets essentially never pass the cuts, all of the monojet events were assumed ${ }^{35}$ to arise from

$$
q g \rightarrow \tilde{q} \tilde{g}
$$

This gives a relatively narrow distribution, but one that is spread out by the recoil $\tilde{g}$. It is also necessary to include the $\tilde{g}$ in the proton when it is so light; this gives additional 
events from

$$
q \tilde{g} \rightarrow \tilde{q} \rightarrow q \tilde{\gamma}
$$

and there is a possible problem from getting too many monojet events from the two mechanisms. Further, for every monojet generated by this latter mechanism, there are $50-60$ jet-jet pairs with an effective mass of $\tilde{M}_{q}$; the $\tilde{q}$ width is $8-10 \mathrm{GeV}$, comparable with the experimental resolution, so this may be an observable peak. Our calculations indicate ${ }^{7}$ about $500 j j$ events from this source, with $M_{j j}$ about $100 \mathrm{GeV}$. It is not clear whether such a signal would be observed.

\section{COMMENTS}

In this discussion of the anomalous events, I have first given a general analysis of how to approach the study of new (possible) experimental phenomena that might take us beyond the Standard Model and I have discussed the constraints on ideas. Then I gave a short review of the kinds of anomalous events expected if supersymmetric partners were being produced. It is very interesting that at the present time the only candidates for anomalous events are the kind that are expected if partners of quarks and gluons are produced. Indeed, other possible kinds of events, which could not have been explained by supersymmetry, have tended to disappear as the data have improved. I have not considered at all whether the anomalous events constitute a signal of phenomena not interpretable within the Standard Model; that is up to the experimenters, who have now proposed such status for the monojets.

Given that situation, it is next necessary to examine whether supersymmetry can quantitatively interpret the data; in this case, that means mainly the UAl monojet data (the situation with dijets plus missing momentum is somewhat unclear experimentally and also unclear theoretically because of the possibility of QCD radiation). It would be of extraordinary significance if new phenomena were discovered at all and of even greater significance if the new phenomena were related to supersymmetry which has such potential theoretical value. It does indeed seem to be the case that at present, there is at least one valid supersymmetric interpretation of the data. Finally, I have discussed choosing among different supersymmetric interpretations (i.e., choices of superpartner masses), which is of importance particularly to workers within the field and to model builders. Further discussion along these lines is available in references 7 , 25,26 , and $31-35$ and in several papers that are surely in preparation by various authors.

\section{ACKNOWLEDGMENTS}

I appreciate a number of useful conversations with V. Barger, S. Glashow, L. Hall, I. Hinchliffe, A. Kernan, M. Mohammadi, F. Paige, and V. Vuillemin. I would like to thank M. Block and his family and Ruth Goldhor for their hospitality and their very effective efforts to make this meeting successful. 


\section{NOTES AND REFERENCES}

1. For other reviews, see HaLL, L. J., R. L. JAFFE \& J. RosNer. 1984. Proceedings of the Workshop on Electroweak Symmetry Breaking, June 1984. LBL-18571; BARGER, V. 1984. Invited talk at Santa Fe DPF Meeting, October 1984, MAD/Ph/229.

2. FNAl Collaboration. Brookhaven, Columbia. To be published; Baltay, C. Private communication.

3. See the talks of Mohammad, M. \& J. Rohlf, UA1, at this conference; see the talk of HANSEN, J., UA2, at this conference.

4. For a review, see Haber, H. E. \& G. L. KANE. 1985. Phys. Rep. 117: 75.

5. Ellis, J. \& H. KowalsKI. 1984. Phys. Lett. 142B: 441.

6. Reya, E. \& D. P. Roy. 1984. Phys. Rev. Lett. 53: 881.

7. BARNETT, R. M., H. E. HABER \& G. L. KANE. 1985. Implications of a systematic study of the CERN monojets for supersymmetry (LBL-18900/SLAC-PUB-3551). Phys. Rev. Lett. 54; Preprint LBL-20102, Aug. 1985.

8. Barger, V., H. Baer \& K. Hagiwara. 1984. Phys. Rev. Lett. 146B: 257.

9. Dusedau, D., D. LuSt \& D. ZePperfeld. 1984. Phys. Lett. 148B: 234.

10. This has been emphasized by Glashow, S. L. \& A. Manohar. 1985. Phys. Rev. Lett. 54: 526.

11. For general constraints from any $S U(2) \times U(1)$ current, see DUNCAN, M. J. \& M. Veltman. 1984. Phys. Lett. 139B: 310.

12. Paige, F. Private communication.

13. KANE, G. L. \& L. MaIANi. 1984. Phys. Lett. 147B: 469.

14. Behrend, H. J. et al. CELlo Collaboration. 1984. Phys. Lett. 141B: 145.

15. Veltman, M. 1984. Phys. Lett. 139B: 139.

16. Hikasa, K-I. 1984. Phys. Lett. 145B: 139 (UT-433, May 1984).

17. KraUss, L. M. 1984. Phys. Lett. 143B: 248.

18. Gronau, M. \& J. Rosner. 1984. Phys. Lett. 147B: 217.

19. HOLDOM, R. 1984. Toronto preprint.

20. Moffat, J. Toronto preprint; KING, S. F. 1985. Phys. Rev. Lett. 54: 528.

21. Weinberg, S. 1976. Phys. Rev. D13: 974; Susskind, L. 1979. Phys. Rev. D20: 2619; Dimopoulos, S. \& L. Susskind. 1979. Nucl. Phys. B155: 237; EICHTEN, E. \& K. LANE. 1980. Phys. Lett. 90B: 125. All numbers are from Dimopoulos, S., S. RabY \& G. L. KANE. 1981. Nucl. Phys. B182: 77.

22. For related signatures, see BERGER, E. L. \& M. JACOB. 1984. Phys. Lett. 147B: 197.

23. Kane, G. L. \& J. P. Leveille. 1982. Phys. Lett. 112B: 227.

24. Campbell, B. A., J. Ellis \& S. Rudaz. 1982. Nucl. Phys. B198: 1; Antoniadis, I., C. Kounnas \& R. Lacaze. 1983. Nucl. Phys. B211: 216; Kounnas, C. \& D. Ross. 1983. Nucl. Phys. B124: 317; Jones, S. K. \& C. H. Llewellyn SMith. 1983. Nucl. Phys. B217: 145; Herrero, M. J., C. Lopez \& F. J. Yndurain. 1984. Nucl. Phys. B244: 207.

25. Herrero, M. J., L. E. Ibanez, C. Lopez \& F. J. Yndurain. 1983. Phys. Lett. 132B: 199; 1984. Phys. Lett. 145B: 430.

26. For further discussion of the possible implications of supersymmetry for other experimental signatures, see HALL, L. J. \& J. POLCHINSKI. Implications of supersymmetric origins for monojets. HUTP-84/AO85; Ellis, J. \& M. SHER, 1984. Phys. Lett. 148B: 309; BAER, H., J. Ellis, D. V. Nanopoulos \& X. Tata. 1984. CERN-TH4059/84; Hall, L. J. Talk at DPF Santa Fe Meeting; and reference 7.

27. Kane, G. L. \& J. P. Leveille. 1982. Phys. Lett. 112B: 227; Harrison, P. R. \& C. H. LleWEllyn SMITH. 1983. Nucl. Phys. B213: 223 (1983. E. B223: 542); ANTONIADIS, I., L. Baulieu \& F. Delduc. 1984. Z. Phys. C23: 119; Dawson, S., E. Eichten \& C. QUIGG. 1984. FERMILAB-PUB-83/82-THY.

28. Eichten, E., I. Hinchliffe, K. Lane \& C. Quigg. 1984. Rev. Mod. Phys. 56: 579.

29. See BARNETT, R. M. \& H. E. Haber. 1984. Simulating supersymmetry at the SSC. To be published in the Proceedings of the 1984 DPF Summer Study on the Design and Utilization of the SSC, Snowmass, Colorado. 
30. ARNISON, G. et al. 1983. Phys. Lett. 132B: 214.

31. ElLIS, J. \& H. Kowalski. 1984. Nucl. Phys. B246: 189.

32. REYA, E. \& D. P. RoY. 1984. Dortmund preprint DO-TH 84/19.

33. BARGER, V., K. Hagiwara, W. Y. KUENG \& J. WoOdSIDE. 1984. Wisconsin preprint MAD/PH/197.

34. Allan, A. R., E. W. N. Glover \& A. D. Martin. 1984. Phys. Lett. 146B: 247; Allan, A. R., E. W. N. Glover \& S. L. GRAYSON. 1984. Durham preprint DTP/84/28.

35. Barger, V., K. Hagiwara \& J. Woodside. 1984. Phys. Rev. Lett. 53: 641; Barger, V., K. Hagiwara \& W. Y. Keung. Phys. Lett. 145B: 147.

36. I appreciate a discussion with $A$. Kernan on this point.

37. Previous analyses are in references $5,31,33$, and 34 . We have carried out our own calculations as described in reference 7, and FIGURE 5 is from reference 7 . 